\title{
A RARE PHENOMENON OF ATYPICAL LIPODYSTROPHY IN A PATIENT ON HAART IN THE ABSENCE OF A PROTEASE INHIBITOR REGIMEN
}

\author{
Mohammed Mitha, $M B C h B$, Dip HIV Man (SA) \\ Gordon Cupido, $B S c, M B C h B, F C P(S A)$ \\ Jantjie Taljaard, $M B C h B$, MMed (Intern), DTM\&H \\ Department of Internal Medicine, Tygerberg Hospital and Stellenbosch University, W Cape
}

\begin{abstract}
Lipodystrophy is a complication of patients on antiretroviral (ARV) medication; however, it is commonest in patients on long-term treatment and those on protease inhibitor $(\mathrm{PI})$ regimens. ${ }^{1{ }^{2}}$ We present a rare case of atypical lipodystrophy, presenting as multiple subcutaneous lipomas, in a patient who had been on a non-PI ARV regimen for 6 weeks.
\end{abstract}

\section{CASE HISTORY}

A 45-year-old man, known to be hypertensive and with a history of smoking (5 cigarettes a day for 18 years), presented to Tygerberg Hospital with peripheral vascular disease necessitating bilateral amputations at the knee. The patient had no other significant medical disorders and no previous history of tuberculosis (TB), and consumed alcohol socially. He had been well before this admission, with no previous medical or surgical admissions. The findings on general and systemic examination were normal and no lumps were noted on the body.

The patient was tested for HIV as part of his surgical work-up and was found to be positive with a CD4 count of 152 cells/ $\mu \mathrm{l}$. He was commenced on ARVs: stavudine (D4T) $30 \mathrm{mg}$ 12-hourly, lamivudine (3TC) 150 $\mathrm{mg}$ 12-hourly and efavirenz (EFV) $600 \mathrm{mg}$ at night. He underwent successful above-knee amputations and was subsequently followed up at the Tygerberg Hospital Infectious Disease Clinic approximately 1 month later.

The patient was doing well and reported no problems or side-effects from the ARVs. The stumps were clean and findings on a thorough physical examination were normal. No lumps were detected on the patient's body at this visit. The patient had no symptoms of $T B$, but a chest radiograph revealed infiltrates in the right upper zone. At this stage we were still awaiting sputum results from his initial admission. Two weeks later (approximately 6 weeks after ARV initiation) drugsusceptible TB was diagnosed on sputum culture. TB treatment was immediately commenced.
The patient returned for a follow-up visit after a further 2 weeks complaining of multiple lumps on the trunk and abdomen. Physical examination revealed multiple soft, mobile, non-tender nodules on the abdomen (Fig. 1), measuring approximately $2 \times 2 \mathrm{~cm}$. A fine-needle aspirate and excision biopsy confirmed that these were lipomas. A fasting lipogram revealed a total cholesterol level of $5.7 \mathrm{mmol} / \mathrm{l}$, a triglyceride level of $2.0 \mathrm{mmol} / \mathrm{l}$, a high-density lipoprotein (HDL) cholesterol level of 1.2 $\mathrm{mmol} / \mathrm{l}$ and a low-density lipoprotein (LDL) cholesterol level of $3.6 \mathrm{mmol} / \mathrm{l}$.

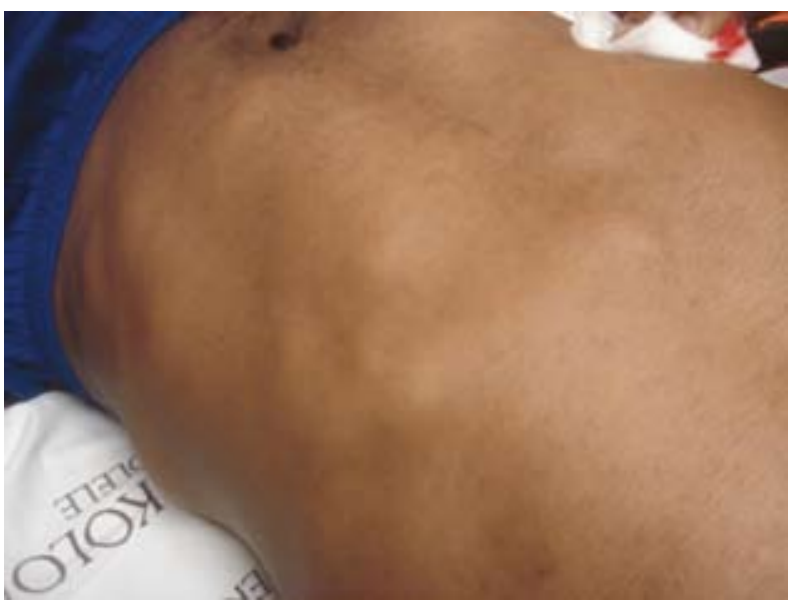

Fig. 1. Multiple lipomas on the patient's abdomen.

The patient was counselled regarding his condition and continued on his anti-TB treatment and ARVs. He was started on the appropriate medical management for his dyslipidaemia and given appropriate dietary advice. 


\section{DISCUSSION}

Lipodystrophy encompasses both lipo-atrophy and lipohypertrophy. Lipo-atrophy presents as subcutaneous fat wasting in the face and peripheries, whereas lipohypertrophy occurs as fat accumulation in the abdomen, breast and dorso-cervical region. ${ }^{2,3}$ Lipodystrophy can be associated with hyperlipidaemia and insulin resistance, which constitute the lipodystrophy syndrome. ${ }^{3}$ The commonest class of ARVs to cause lipodystrophy is the PIs. ${ }^{1-3}$ These agents are commonly used in second-line regimens in ARV treatment in South Africa.

This patient presented with multiple subcutaneous lipomas on the abdomen 6 weeks after commencing ARVs and approximately 2 weeks after starting TB treatment. This was an extremely interesting and yet perplexing presentation. Diagnosis of a novel immune reconstitution inflammatory syndrome phenomenon or a cutaneous lymphoma was considered, but the patient was clinically well, histological examination confirmed a lipoma with no inflammatory cell infiltrate, and biopsies of the lesions did not culture TB or any other bacterial organism.

The reason for the acute onset of the lipomas, and in a patient not on a Pl, eludes the authors. The patient's background medical condition of hypertension and peripheral vascular disease may have contributed to the development of the lipomas. Google Scholar and Pubmed searches in the English language revealed only 1 other case in which subcutaneous lipomas developed on a regimen without Pls. ${ }^{4}$ That patient had been on
3TC, tenofovir and EFV. It is unclear whether EFV, 3TC or both contributed to the lipomas in our case, as that patient was also on both these agents.

Most patients who develop lipodystrophy syndrome while on Pls are switched to a non-nucleoside reverse transcriptase inhibitor, usually with improvement in their dyslipidaemia. ${ }^{4,5}$ Owing to the unique nature of our case, with no evidence base to guide treatment, it was decided to continue the regimen of $3 \mathrm{TC}, \mathrm{D} 4 \mathrm{~T}$ and EFV.

It is important that clinicians are aware of this rare and atypical form of lipodystrophy, which can apparently occur in patients taking nucleoside reverse transcriptase inhibitors and non-nucleoside reverse transcriptase inhibitors. If it occurs, the most important issue is to counsel the patient regarding lipomas and their benign nature and that they should remain compliant on treatment. It would be useful if a registry could be created to document such atypical adverse effects of ARVs in order for research to be conducted which may prevent such phenomena and guide us on appropriate drug changes if and when required.

\section{REFERENCES}

1. Miller J, Carr A, Smith D, et al. Lipodystrophy following antiretroviral therapy of primary HIV infection. AIDS 2000; 15: 2406-2407.

2. Southern African HIV Clinicians Society. Guidelines: Antiretroviral therapy in adults. Southern African Journal of HIV Medicine 2008; 9(1): 18-31.

3. Brown $\Pi$. Approach to the human immunodeficiency virus-infected patient with lipodystrophy. J Clin Endocrinol Metab 2008; 93(8): 2937-2945.

4. Balestreire $E$, Haught JM, English JC. Multiple subcutaneous lipomas induced by HAART in the absence of protease inhibitors. Arch Dermatol 2007; 143(12): 15961597.

5. Mallewa JE, Wilkens E, Vilar J, et al. HIV-associated lipodystrophy: a review of underlying mechanisms and therapeutic options. J Antimicrob Chemother 2008; 62(4): 648-660.

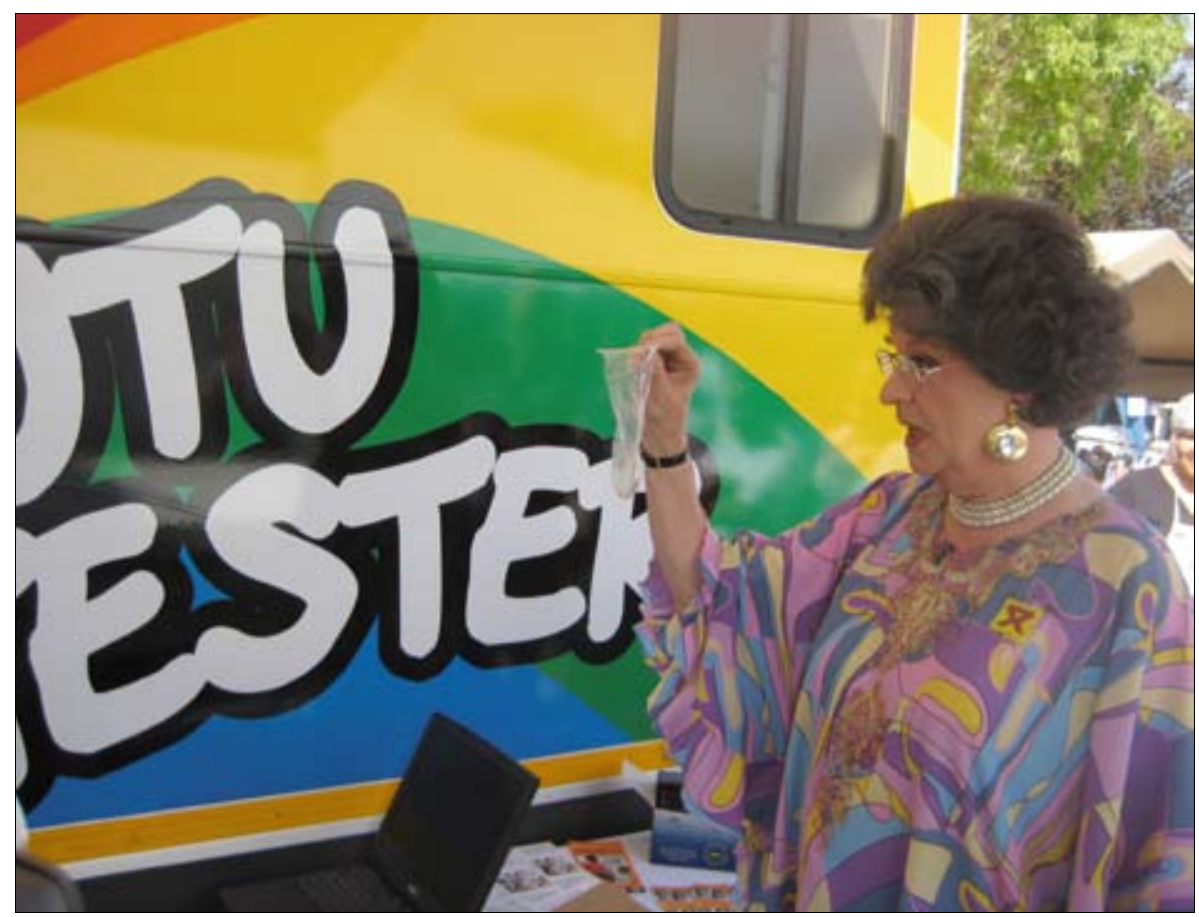

